\title{
Case Report \\ Hemophagocytic Lymphohistiocytosis in Association with Primary Cutaneous Anaplastic Large Cell Lymphoma
}

\author{
Aneesh Basheer, Somanath Padhi, Ramesh Nagarajan, \\ Vinoth Boopathy, Sudhagar Mookkappan, and Nayyar Iqbal \\ Pondicherry Institute of Medical Sciences, Ganapathichettikulam, Pondicherry 605014, India \\ Correspondence should be addressed to Aneesh Basheer; basheeraneesh@gmail.com
}

Received 11 August 2014; Revised 27 September 2014; Accepted 27 September 2014; Published 28 October 2014

Academic Editor: Massimo Gentile

Copyright (c) 2014 Aneesh Basheer et al. This is an open access article distributed under the Creative Commons Attribution License, which permits unrestricted use, distribution, and reproduction in any medium, provided the original work is properly cited.

\begin{abstract}
Hemophagocytic lymphohistiocytosis (HLH) has a well known association with lymphomas, especially of T cell origin. Prognosis of lymphoma associated HLH is very poor, especially in T cell lymphomas; and, therefore, early diagnosis might alter the outcome. Though association of HLH with systemic anaplastic large cell lymphoma (ALCL) is known, its occurrence in primary cutaneous ALCL (C-ALCL) is distinctly rare. We aim to describe a case of C-ALCL (anaplastic lymphoma kinase (ALK)-) in an elderly male who succumbed to the complication of associated $\mathrm{HLH}$, which was possibly triggered by coexistent virus infection. We briefly present the literatures on lymphoma associated HLH and discuss the histopathological differentials of cutaneous CD30+ lymphoproliferative disorders. We do suggest that HLH may pose diagnostic challenges in the evaluation of an underlying lymphoma and hence warrants proper evaluation for the underlying etiologies and/or triggering factors.
\end{abstract}

\section{Introduction}

Hemophagocytic lymphohistiocytosis (HLH) is a rare, potentially underrecognized, hyperinflammatory syndrome that may be primary or secondary to several conditions such as infections (most common), inflammatory disorders, and hematological malignancies. Epstein Barr virus (EBV) is reported to be the most common risk factor implicated in the pathogenesis of HLH $[1,2]$. Lymphoma associated HLH is the most common form of malignancy associated HLH [3]. Although HLH is known to complicate several subtypes of lymphomas, its association with $\mathrm{T}$ cell non-Hodgkin lymphoma (T-NHL) is reported to be most common; and occurrence among patients with B cell NHL (B-NHL), and Hodgkin lymphoma (HL) is rare [3-5].

Anaplastic large cell lymphoma (ALCL) is an uncommon form of mature $\mathrm{T}$ cell lymphoma which constitutes 2 to $3 \%$ of all NHLs. It is characterized by a polymorphous population of both neoplastic large pleomorphic cells (with an "embryo-like" or "horse-shoe" like nuclei (so-called Hallmark cell)) and reactive inflammatory cells, a tendency to invade lymphoid sinuses, and widespread expression of CD30 antigen (in greater than $75 \%$ of the tumor cells). Majority of these tumors are associated with a specific translocation $t(2 ; 5)(\mathrm{p} 23$; q35) resulting in the overexpression of the anaplastic lymphoma kinase (ALK) gene [6]. As per the 2008 WHO classification, ALCL encompasses 3 entities with overlapping histopathologic, but contrasting clinical and immunophenotypic characteristics, namely, systemic ALCL (ALK+), systemic ALCL (ALK-), and primary cutaneous ALCL (C-ALCL) [7]. There have been sporadic reports of HLH complicating the clinical course of ALCL, more so in systemic form of ALCL, rather than C-ALCL $[8,9]$.

In this report, we aim to describe a case of C-ALCL in an elderly male who succumbed to the complication of coexisting HLH, possibly triggered by underlying viral infection. Furthermore, we present a brief literature review on lymphoma associated HLH and discuss the diagnostic challenges in evaluation of coexistent HLH and lymphoma.

\section{Case Presentation}

A 56-year-old farmer presented to the out-patient department with complaints of high grade, intermittent fever, abdominal distension, and vomiting for 20 days. He also 


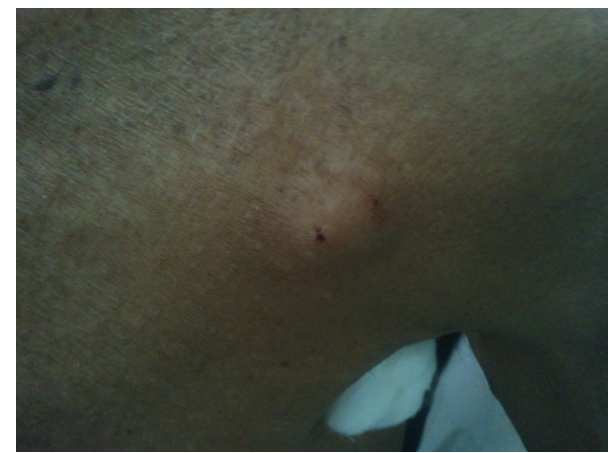

FIGURE 1: Swelling measuring $2 \times 2 \mathrm{~cm}$ over the left shoulder. Swelling was hard and non-tender.

suffered from loss of appetite for past 5 days. He noticed a swelling over his left shoulder since past 5 days. There was no history of loss of weight, breathlessness, cough, or bone pain. Ten months prior to his present admission, he was evaluated in the same hospital for fever and jaundice and found to have a transudative pleural effusion and acalculous cholecystitis. Subsequently, he underwent laparoscopic cholecystectomy. He neither smoked nor consumed alcohol.

On examination, he was febrile. General physical examination revealed pallor, pan-digital clubbing, and bipedal edema. Besides, there were 2 nontender, hard, subcutaneous swellings, one measuring $2 \times 2 \mathrm{~cm}$ on the left shoulder and the other $1 \times 1 \mathrm{~cm}$ over the left anterior axillary line (Figure 1 ). Systemic evaluation revealed firm, nontender hepatomegaly (liver span, $16 \mathrm{~cm}$ ) and firm splenomegaly ( $4 \mathrm{~cm}$ below the left costal margin), and no significant peripheral lymphadenopathy. There were no stigmata of chronic liver disease. Disseminated tuberculosis, human immunodeficiency virus (HIV) infection, disseminated malignancy (lymphomas/leukemia), and connective tissue disorders were among the differential diagnoses considered.

Laboratory investigations showed normocytic normochromic anemia (hemoglobin (Hb), $84 \mathrm{~g} / \mathrm{L}$ (reference, 120-140 g/L)), leukopenia (total leucocyte count (TLC), $2 \times$ $10^{9} / \mathrm{L}$ (reference, $\left.4-11 \times 10^{9} / \mathrm{L}\right)$ ), differential of neutrophil, $49 \%$, lymphocyte, 49\%, and monocytes, 2\%, absolute neutrophil count (ANC), $980 / \mathrm{cmm}$, total platelet count (TPC), $175 \times 10^{9} / \mathrm{L}$ (reference, $150-450 \times 10^{9} / \mathrm{L}$ ), and no atypical cells/blasts. His biochemical parameters revealed elevated serum aspartate aminotransferase/SGOT (546 U/L, reference, 15-41 U/L), alanine aminotransferase/SGPT (208 U/L, reference, 10-40 U/L), alkaline phosphatase (701 U/L, reference, 38-126 U/L), lactate dehydrogenase $(1094 \mathrm{U} / \mathrm{L}$, reference, <300 U/L), ferritin $(2400 \mathrm{ng} / \mathrm{mL}$, reference, $25-340 \mathrm{ng} / \mathrm{mL})$, and vitamin $B_{12}(>2000 \mathrm{pg} / \mathrm{mL}$, reference, $191-890 \mathrm{pg} / \mathrm{mL}$ ). His complete microbiological work-up for possible infectious organisms and serologic tests for HIV, HBV, HCV, ANA, and antidouble stranded DNA were negative. Complete radiological evaluation revealed hepatosplenomegaly (without any focal lesions), mild ascites, and bilateral pleural effusion.

Patient continued to have spiking fever, and, in view of persistent bicytopenia, bone marrow aspiration and biopsy were done, which showed increased population of benign histiocytes with evidence of hemophagocytosis. There was no evidence of any granuloma, hemoparasites, or malignancy. Histopathological evaluation of the excised left shoulder nodule revealed a high grade tumor in the dermis with large, nonepidermotropic, pleomorphic cells with "horseshoe" shaped nuclei and prominent nucleoli. There was evidence of vascular proliferation and the tumor cells exhibited angiocentricity. Further characterization using immunohistochemistry (IHC) showed that the tumor cells were positive for leucocyte common antigen (LCA), CD3, and CD30 (uniform diffuse positivity in Golgi region in more than $75 \%$ of cells) but negative for pan cytokeratin, CD20, ALK, and epithelial membrane antigen (EMA). The tumor cells showed a higher proliferation index $(\mathrm{Ki}-67=70 \%)$. The histopathological features, in correlation with IHC characteristics, were consistent with the diagnosis of ALCL (CD30+, ALK-) (Figures 2(a), 2(b), 2(c), 3(a), 3(b), 4(a), 4(b), 4(c), and $4(\mathrm{~d}))$. In view of the domainant cutaneous presentation, lack of significant lymphadenopathy and absence of obvious bone marrow infiltration (CD 30 negative), the diagnosis of C-ALCL with associated HLH at presentation was rendered. The patient was managed with steroids and ongoing broad spectrum intravenous antibiotics, and hematologist's opinion was taken. However, patient succumbed to disseminated intravascular coagulation (DIC) and multiorgan dysfunction, secondary to HLH, on 5th day of diagnosis of the HLH and lymphoma (11th day post admission). The informed consent was obtained from the next of kin of the patient.

\section{Discussion}

The hallmark of HLH is phagocytosis of mature blood elements (red cells, leucocytes, and platelets) or their precursors by benign macrophages throughout the reticuloendothelial system secondary to immune dysregulation and excessive triggering of cytokine production. In the absence of a positive family history or a documented genetic/molecular abnormality, the diagnosis of $\mathrm{HLH}$ requires the presence of at least, five of eight HLH-2004 criteria put forth by the HLH society [10]. The diagnosis of lymphoma associated HLH requires the same criteria in addition to the documented histological evidence of a lymphoma [3]. Our case satisfied the required criteria (5/6 tested) for the diagnosis of $\mathrm{HLH}$ such as persistent high grade fever, bicytopenia $(\mathrm{Hb}<90 \mathrm{~g} / \mathrm{L}$, ANC $<1000 / \mathrm{cmm})$, splenomegaly in the absence of focal lesions, hyperferritinemia, and histiocytic hemophagocytosis in the bone marrow. In addition to HLH, there was a documented evidence of a lymphoma in our patient.

A comprehensive review on lymphoma associated HLH is presented in Table 1. Compared to B cell NHLs and HLs, $\mathrm{T}$ cell NHLs are frequently reported to be complicated with HLH during their course of the disease; and this confers a worse prognosis $[4,5,11-14]$. Lymphomas associated with $\mathrm{HLH}$ are more often reported among males than females. The median age at presentation/diagnosis for T-NHL and HL associated HLH has been reported to be 45 years, whereas BNHL associated HLH has been frequently reported among 


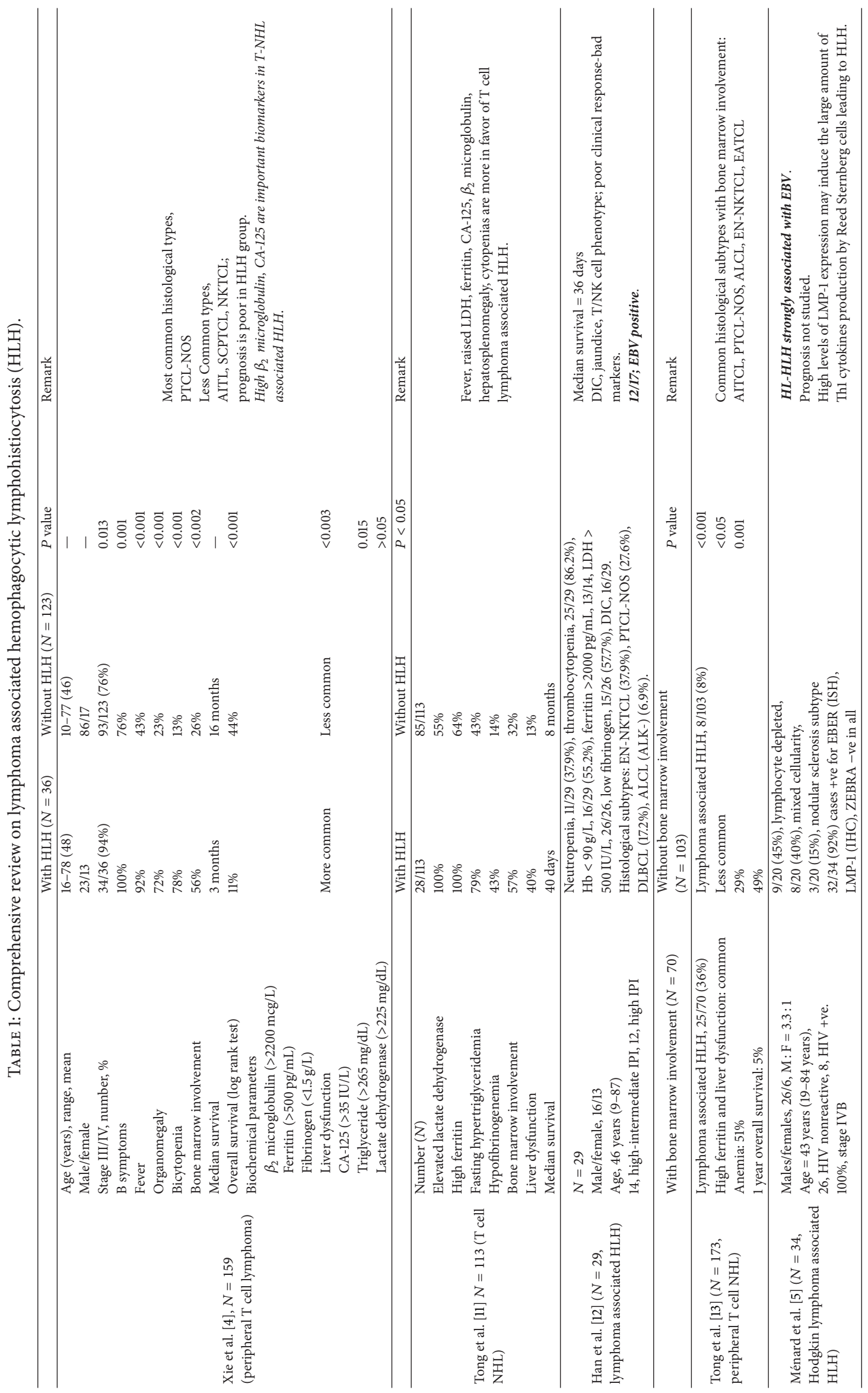




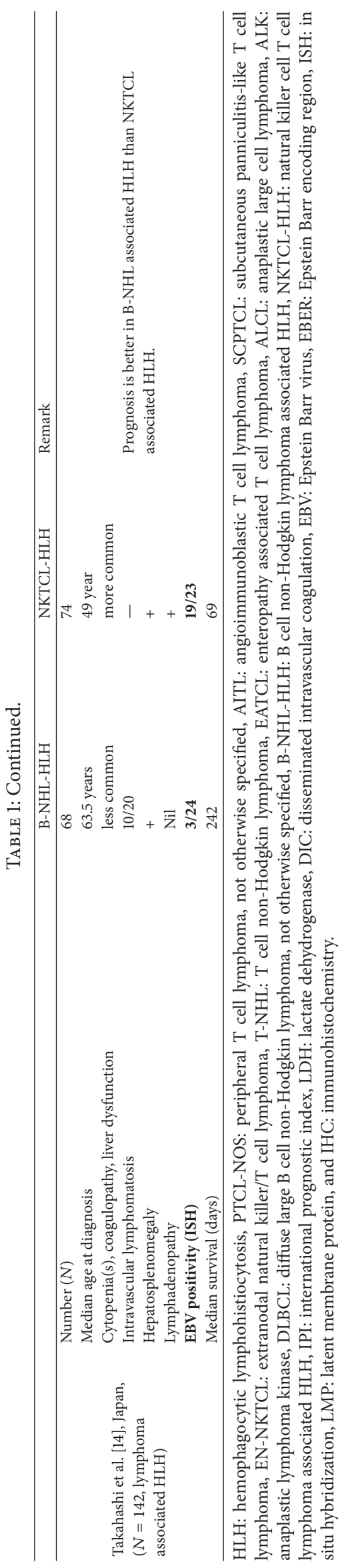




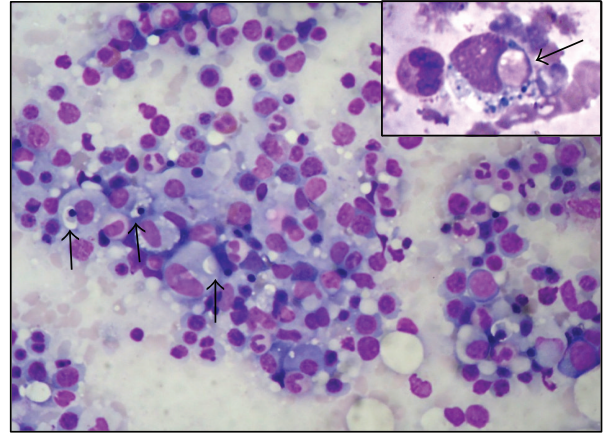

(a)

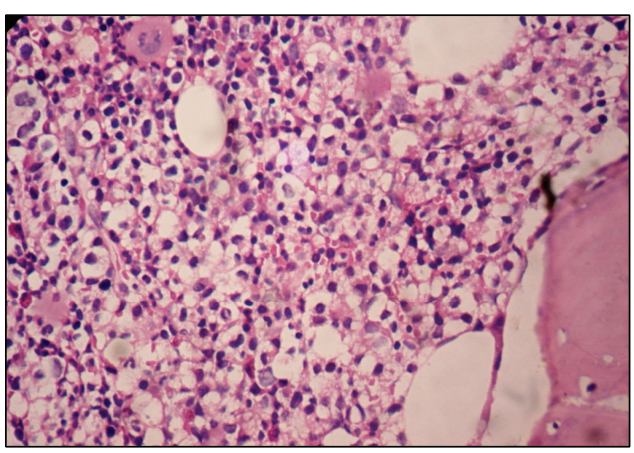

(b)

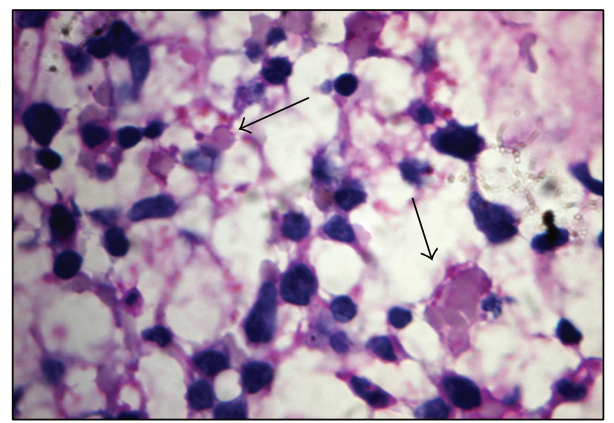

(c)

FIGURE 2: Bone marrow aspirate smear (a) showing hypercellularity and increased population of benign histiocytes with evidence of haemophagocytosis (black arrows, 200x). Note the evidence of erythrophagocytosis by the histiocytes (inbox, black arrow, 400x) (May Grunewald Giemsa stain). Bone marrow trephine biopsy section showing increased population of benign histiocytes in the intertrabecular marrow space (b, haematoxylin eosin, 200x). These histiocytes showed engulfed debris and obvious erythrophagocytosis (c, black arrow) (haematoxylin eosin, 400x).

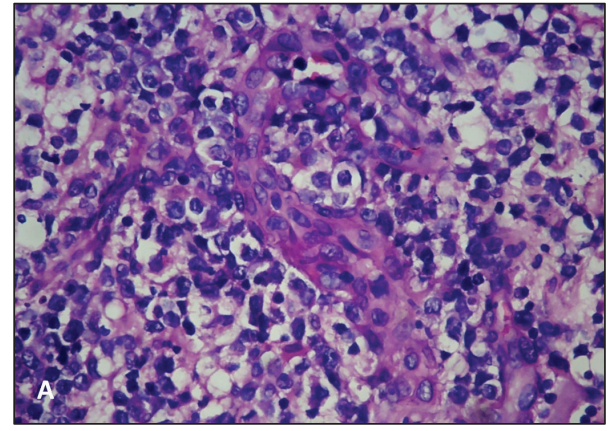

(a)

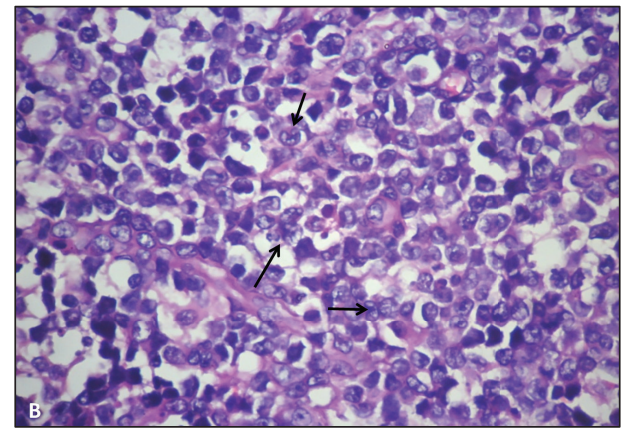

(b)

FIGURE 3: Paraffin embedded tissue sections from the excised left shoulder nodule showing sheets and aggregates of pleomorphic tumor cells with irregular nuclear contour and prominent nucleoli. The tumor cells showed angiocentricity (a) and at places, "embryo"/"horse shoe" like nuclei, prominent nucleoli (so-called "hallmark" cells) ((b), black arrows) (haematoxylin eosin, 400x).

elderly population (median age $=6$ th decade) $[3,12,14]$. Peripheral T cell lymphoma, not otherwise specified (PTCLNOS), extranodal NK/T cell lymphomas (EN-NKTCL), subcutaneous panniculitis-like $\mathrm{T}$ cell lymphoma (SCPTCL), angioimmunoblastic $\mathrm{T}$ cell lymphoma (AITL), and rarely ALCL are more likely to be complicated by secondary HLH $[4,7,11-14]$. Among B-NHLs, diffuse large B cell lymphoma (DLBCL) is the most common histological subtype implicated in HLH. Among HL, lymphocyte depleted and mixed cellularity subtypes have been reported to be associated with HLH [5]. Several large population based studies among Asian patients have found that T-NHL associated HLH is characterized by (i) advanced stage at presentation, (ii) frequent occurrence of B symptoms, organomegaly (and lymphadenopathy), cytopenia (s), liver dysfunction, and coagulopathy, (iii) higher proportion of bone marrow infiltration, and (iv) markedly reduced overall survival with or without definitive chemotherapy. Several biochemical 


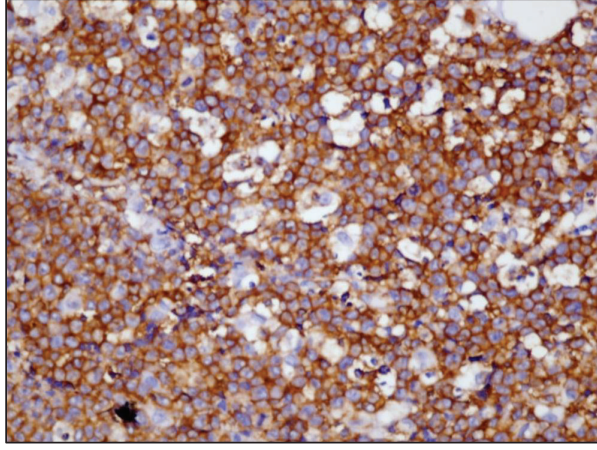

(a)

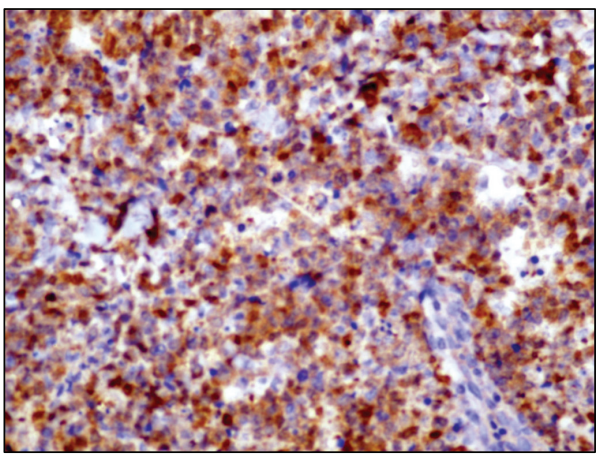

(c)

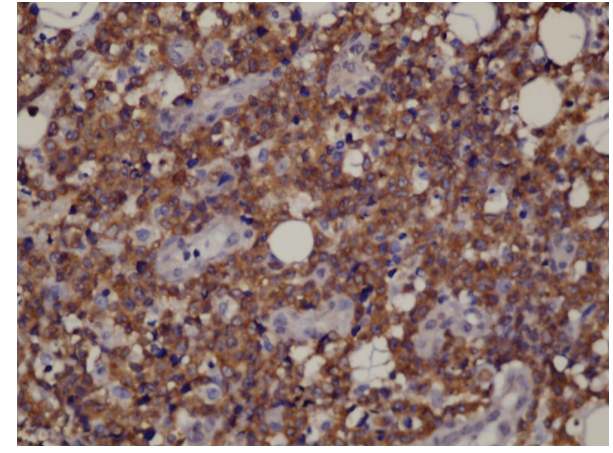

(b)

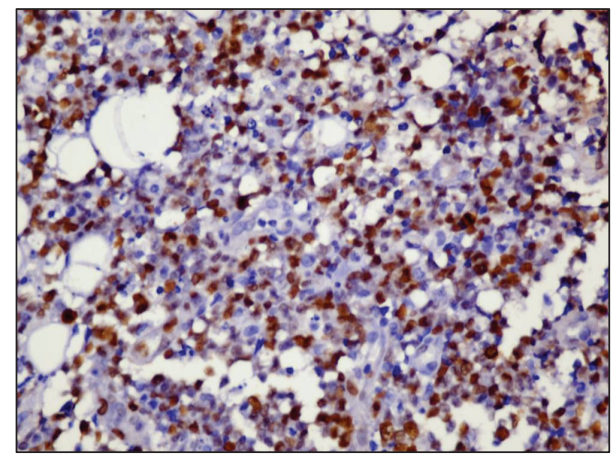

(d)

FIGURE 4: On immunohistochemistry the tumor cells showed uniform intense positivity for leukocyte common antigen (LCA) (a), CD3 (b), and CD30 (c), with a high proliferation index (Ki-67 = 70\%) (d). Note the uniform, strong Golgi region positivity of CD30 (c) in the majority (>75\%) of tumor cells. The cells were negative for pancytokeratin, CD20, anaplastic large cell lymphoma kinase (ALK), and epithelial membrane antigen (EMA) (Peroxidase-antiperoxidase stain, a and b; 100x, c; 200x).

parameters such as raised $\beta_{2}$ microglobulin $(>2200 \mathrm{mcg} / \mathrm{L})$, CA-125 ( $>35 \mathrm{IU} / \mathrm{L}$ ), ferritin, fasting triglyceride, and lactate dehydrogenase and reduced fibrinogen have been reported to be significantly $(P<0.001)$ associated with HLH subgroups of T-NHL and have been suggested to be important biomarkers of T-NHL associated HLH [4, 11-13]. On the contrary, B-NHL associated HLH has been reported to be associated with lesser degree of bone marrow involvement, significant lack of associated lymphadenopathy, a higher propensity for intravascular lymphomatosis, higher association with EBV, and overall better survival following chemotherapy $[3,14]$. The largest French study (to date) on HL associated HLH $(N=34,8 / 34 ; \mathrm{HIV}+\mathrm{ve})$ reported that $100 \%$ of these patients were at advanced stage (IVB), and there was a strong association $(32 / 34,92 \%)$ with EBV detected by either immunohistochemistry or in situ hybridization technique [5]. The authors of that study suggested that high levels of latent membrane protein (LMP)-1 expression may induce the large amount of $\mathrm{Th}_{1}$ cytokines production by Reed Sternberg cells in HL leading to HLH.

Primary cutaneous ALCL is the second most common form of primary cutaneous $\mathrm{T}$ cell lymphoma after mycoses fungoides and carries an excellent prognosis with 5-year overall survival of nearly $90 \%$ following surgical excision and/or local radiation therapy. Though it presents as a solitary ulcerated nodule, multifocal involvement may be seen in less than $20 \%$ of cases. Along with benign/reactive lymphomatoid papulosis (LyP), it constitutes the spectrum of primary CD30 positive lymphoproliferative disorders of the skin. However, this needs to be differentiated from the more common cutaneous involvement in both PTCLNOS and systemic ALCL (ALK-) by means of appropriate ancillary studies $[6,7]$. Simultaneous occurrence of HLH and lymphoma in our patient led to a diagnostic dilemma with more common PTCL-NOS and/or systemic ALCL (ALK-) with dominant cutaneous involvement. However, our patient had following characteristics which favored a final diagnosis of C-ALCL over systemic ALCL (ALK-)/PTCL-NOS. These include (i) older age at presentation, (ii) dominant cutaneous involvement in the absence of demonstrable involvement of bone marrow, (iii) lack of significant peripheral lymphadenopathy, and (iv) ALK negative, intense, and Golgi zone CD30 positivity in $>75 \%$ tumor cells (in contrast to focal positivity in PTCL-NOS) [7]. Furthermore, only few specific markers such as nuclear survivin expression (absent in CALCL, positive in systemic ALCL) and punctuate clusterin expression (seen in C-ALCL) may help in differentiating C-ALCL from systemic ALCL with dominant cutaneous involvement [6]. These markers were not used in our case due to non availability in our center. Considering the favorable outcome in C-ALCL compared to its systemic counterpart, we presume that the fatal outcome in our patient was more 
likely the result of complication of HLH, rather than the CALCL itself. This probably was the result of an underlying triggering event such as infection with EBV, Parvo-B19, or cytomegalo virus which unfortunately was not tested in our patient due to financial and time constraint.

To conclude, dominant presentation of HLH may overshadow the underlying lymphoma creating great diagnostic and therapeutic challenges for the treating physician. Therefore, a high index of suspicion, early diagnosis, and early institution of therapy might alter the outcome in these patients.

\section{Conflict of Interests}

The authors declare that there is no conflict of interests regarding the publication of this paper.

\section{References}

[1] G. N. Usmani, B. A. Woda, and P. E. Newburger, "Advances in understanding the pathogenesis of HLH," The British Journal of Haematology, vol. 161, no. 5, pp. 609-622, 2013.

[2] S. F. N. Bode, K. Lehmberg, A. Maul-Pavicic et al., "Recent advances in the diagnosis and treatment of hemophagocytic lymphohistiocytosis," Arthritis Research and Therapy, vol. 14, no. 3, article 213, 2012.

[3] I. Tzannou, A. M. Balta, and M. Bakiri, "Hemophagocytic syndrome associated with hematologic malignancies," Hospital Chronicles, vol. 6, no. 3, pp. 110-117, 2011.

[4] W. Xie, K. Hu, F. Xu et al., "Clinical analysis and prognostic significance of lymphoma-associated hemophagocytosis in peripheral T cell lymphoma," Annals of Hematology, vol. 92, no. 4, pp. 481-486, 2013.

[5] F. Ménard, C. Besson, P. Rincé et al., "Hodgkin lymphomaassociated hemophagocytic syndrome: a disorder strongly correlated with Epstein-Barr virus," Clinical Infectious Diseases, vol. 47, no. 4, pp. 531-534, 2008.

[6] M. C. Kinney, R. A. Higgins, and E. A. Medina, "Anaplastic large cell lymphoma: twenty-five years of discovery," Archives of Pathology and Laboratory Medicine, vol. 135, no. 1, pp. 19-43, 2011.

[7] S. H. Swerdlow, E. Campo, N. L. Harris et al., WHO Classification of Tumours of Hematopoietic and Lymphoid Tissues, International Agency for Research on Cancer, Lyon, France, 4th edition, 2008.

[8] Y. Shimizu, K. Tanae, N. Takahashi et al., "Primary cutaneous anaplastic large-cell lymphoma presenting with hemophagocytic syndrome: a case report and review of the literature," Leukemia Research, vol. 34, no. 2, pp. 263-266, 2010.

[9] W. Balwierz, M. Czogała, and E. Czepko, "Anaplastic large cell lymphoma associated with hemophagocytic lymphohistiocytosis: a case report and review of the literature," Przeglą, lekarski, vol. 67, no. 6, pp. 436-438, 2010.

[10] J.-I. Henter, A. Horne, M. Aricó et al., "HLH-2004: diagnostic and therapeutic guidelines for hemophagocytic lymphohistiocytosis," Pediatric Blood and Cancer, vol. 48, no. 2, pp. 124-131, 2007.

[11] H. Tong, Y. Ren, H. Liu et al., "Clinical characteristics of T-cell lymphoma associated with hemophagocytic syndrome: comparison of T-cell lymphoma with and without hemophagocytic syndrome," Leukemia and Lymphoma, vol. 49, no. 1, pp. 81-87, 2008.

[12] A.-R. Han, H. R. Lee, B.-B. Park et al., "Lymphoma-associated hemophagocytic syndrome: clinical features and treatment outcome," Annals of Hematology, vol. 86, no. 7, pp. 493-498, 2007.

[13] H. Tong, Y. Ren, W. Qian et al., "Clinicopathological study on peripheral T-cell non-Hodgkin lymphoma with bone marrow involvement: a retrospective analysis from China," International Journal of Hematology, vol. 90, no. 3, pp. 303-310, 2009.

[14] N. Takahashi, A. Chubachi, I. Miura, S. Nakamura, and A. B. Miura, "Lymphoma-associated hemophagocytic syndrome in Japan," Rinsho Ketsueki, vol. 40, no. 7, pp. 542-549, 1999. 


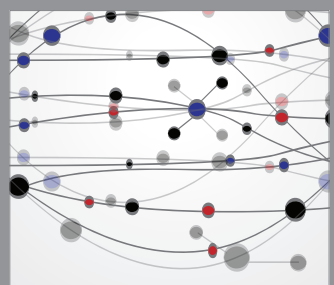

The Scientific World Journal
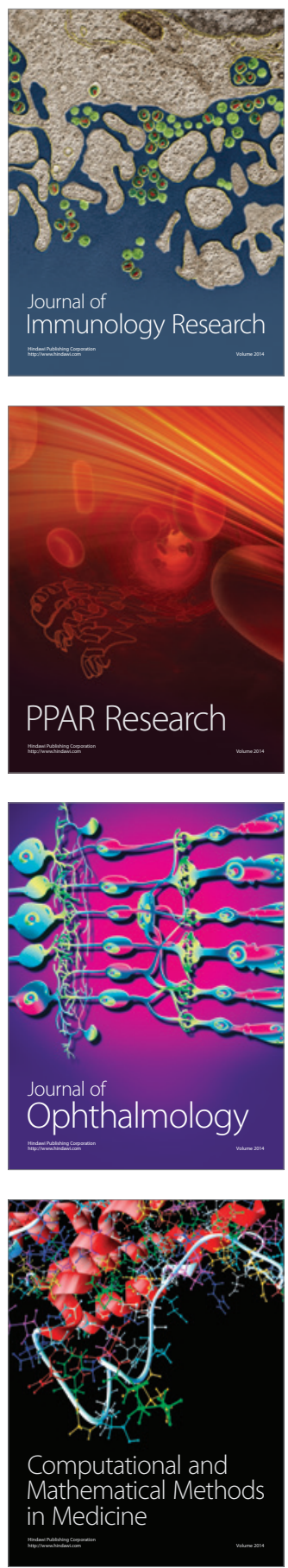

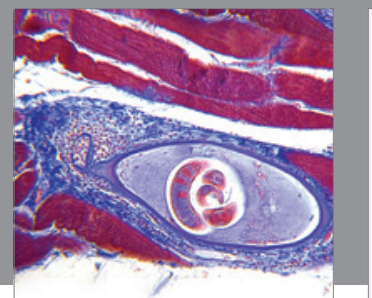

Gastroenterology

Research and Practice
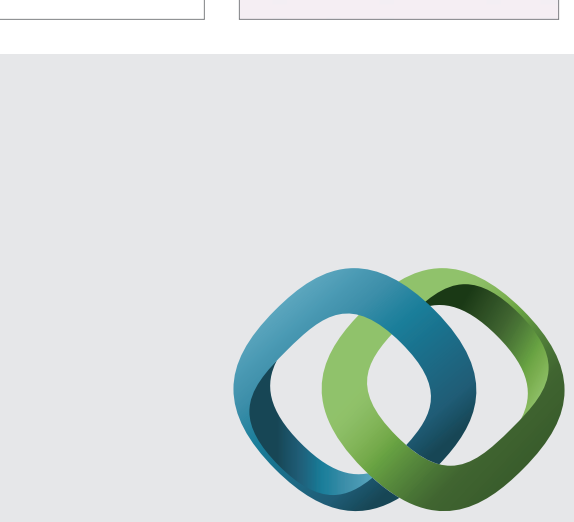

\section{Hindawi}

Submit your manuscripts at

http://www.hindawi.com
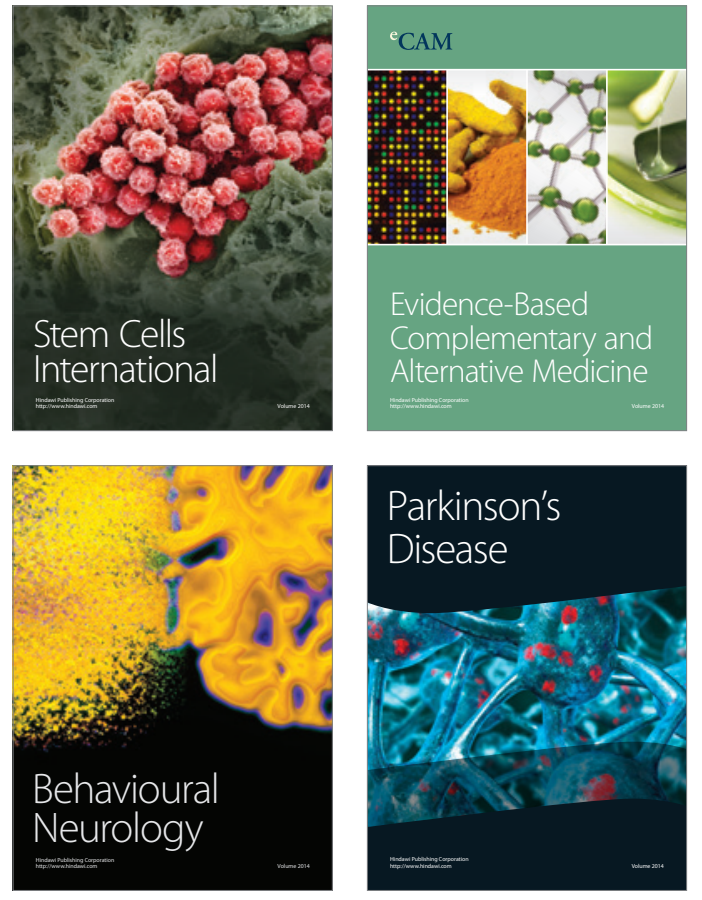
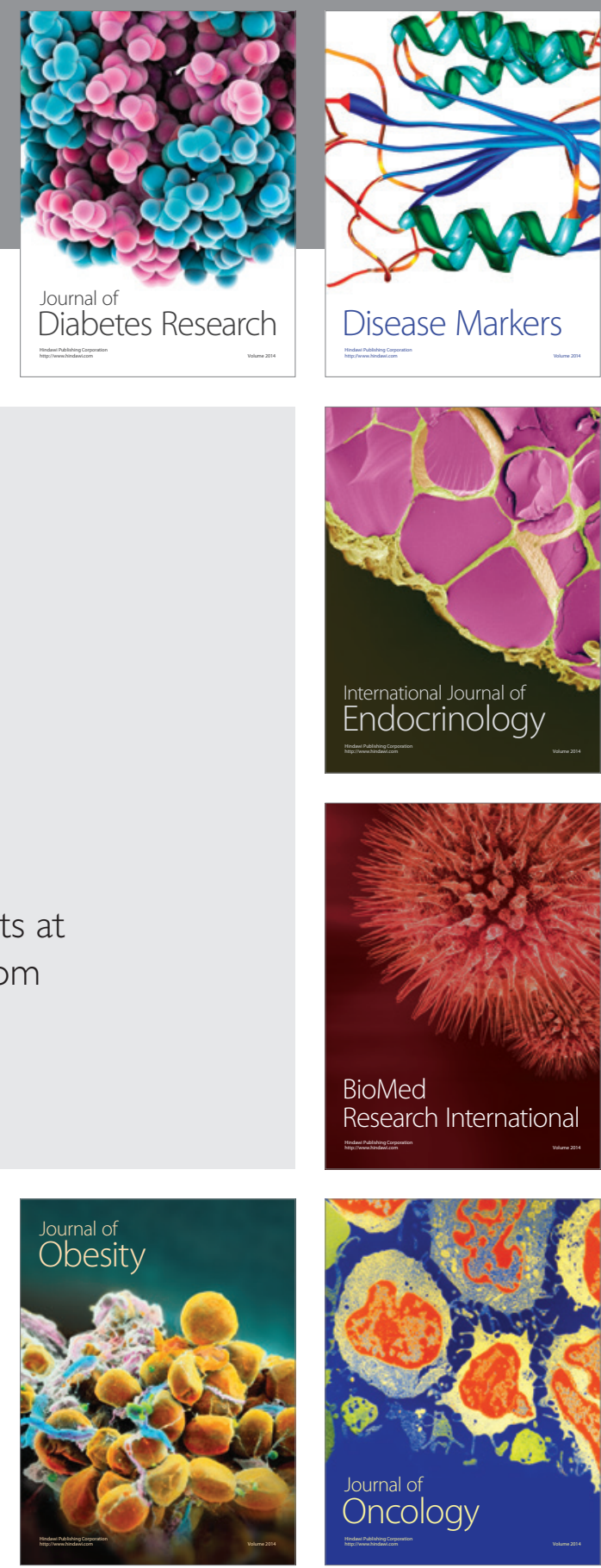

Disease Markers
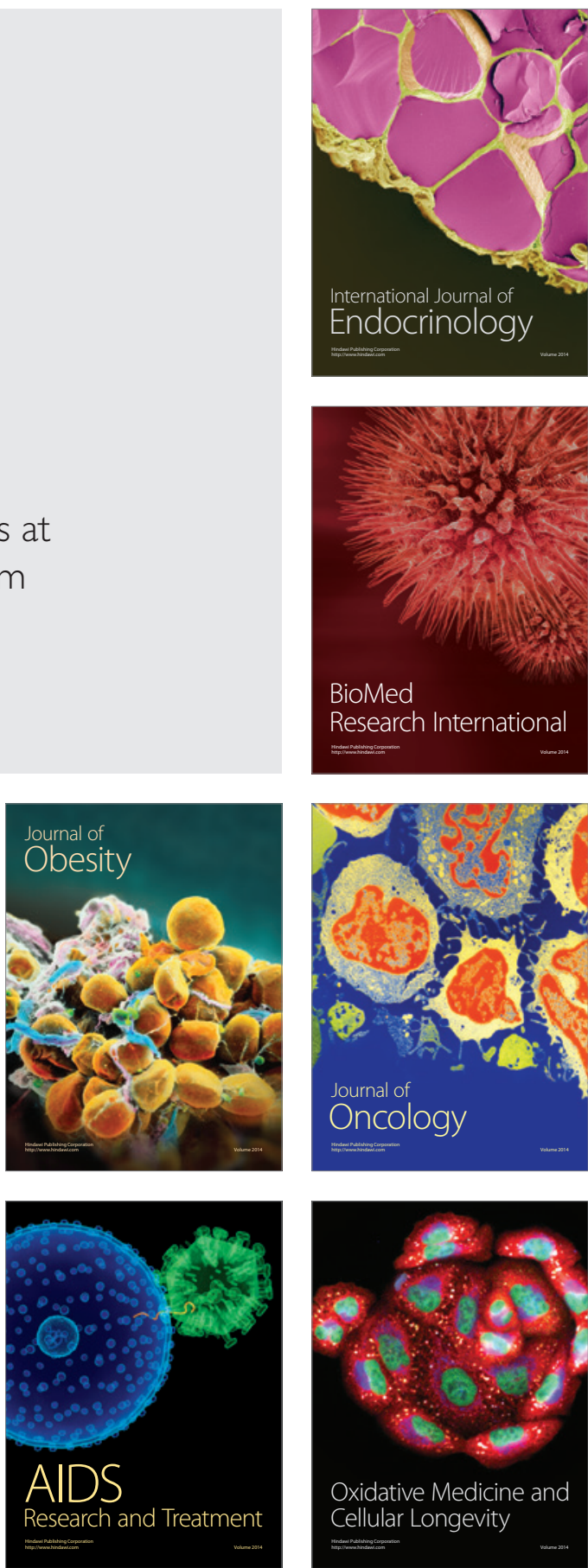